\title{
Quadratically many colorful simplices
}

\author{
IMRE BÁRÁNY* \\ Rényi Institute of Mathematics \\ Hungarian Academy of Sciences \\ POBox 127, 1364 Budapest, Hungary \\ and \\ Department of Mathematics \\ University College London \\ Gower Street, London WC1E 6BT \\ England

\section{JiŘí MATOUŠEK} \\ Department of Applied Mathematics and \\ Institute of Theoretical Computer Science (ITI) \\ Charles University \\ Malostranské nám. 25, 11800 Praha 1 \\ Czech Republic
}

Revised Oct/6/2005 JM

\begin{abstract}
The colorful Carathéodory theorem asserts that if $X_{1}, X_{2}, \ldots, X_{d+1}$ are sets in $\mathbf{R}^{d}$, each containing the origin 0 in its convex hull, then exists a set $S \subseteq X_{1} \cup \cdots \cup X_{d+1}$ with $\left|S \cap X_{i}\right|=1$ for all $i=1,2, \ldots, d+1$ and $0 \in \operatorname{conv}(S)$ (we call $\operatorname{conv}(S)$ a colorful covering simplex). Deza, Huang, Stephen and Terlaky proved that if the $X_{i}$ are in general position with respect to 0 (consequently, each $X_{i}$ has at least $d+1$ points), then there are at least $2 d$ colorful covering simplices, and they constructed an example with no more than $d^{2}+1$ such simplices. Under the same assumption, we show that there are at least $\frac{1}{5} d(d+1)$ colorful covering simplices, thus determining the order of magnitude. We also obtain a lower bound of $3 d$, which is better for small $d$, and in particular, together with a parity argument it settles the case $d=3$, where the minimum possible number of colorful covering simplices is 10 .
\end{abstract}

\section{Introduction}

The following theorem, proved by the first author [1], has found numerous applications (see [2] and [3]):

Theorem 1.1 (Colorful Carathéodory theorem) Let $X_{1}, X_{2}, \ldots, X_{d+1}$ be finite sets in $\mathbf{R}^{d}$ such that $0 \in \operatorname{conv}\left(X_{i}\right)$ for all $i=1,2, \ldots, d+1$. Then there exists a $(d+1)$-point set $S \subseteq X_{1} \cup \cdots \cup X_{d+1}$ with $\left|X_{i} \cap S\right|=1$ for each $i$ and such that $0 \in \operatorname{conv}(S)$.

\footnotetext{
${ }^{*}$ Supported by Hungarian National Foundation Grants T 046246 and T 037846.
} 
If we imagine that the points of $X_{i}$ have color $i$, then the theorem asserts the existence of a colorful set $S$ with $0 \in \operatorname{conv}(S)$, where "colorful" means "containing all colors". We call the convex hull of such an $S$ a colorful covering simplex.

We will assume throughout this paper that the sets $X_{i}$ as in the colorful Carathéodory theorem are in general position with respect to 0 , meaning that $X_{i} \cap X_{j}=\emptyset$ for $i \neq j$ and no $k+1$ points of $X=X_{1} \cup \cdots \cup X_{d+1}$ lie in a common $k$-dimensional linear subspace of $\mathbf{R}^{d}$, for all $k=0,1, \ldots, d-1$. In this situation $0 \in \operatorname{conv}\left(X_{i}\right)$ implies $\left|X_{i}\right| \geq d+1$.

It was shown in [1] that if the $X_{i}$ are as in the colorful Carathéodory theorem and in general position with respect to 0 , then there are actually at least $d+1$ colorful covering simplices. The minimum possible number of colorful covering simplices was investigated by Deza et al. [4], who improved the lower bound to $2 d$, and on the other hand, they exhibited a configuration with only $d^{2}+1$ colorful covering simplices. They conjectured that this is actually the minimum possible number.

We prove that this is at least the correct order of magnitude.

Theorem 1.2 Let $X_{1}, \ldots, X_{d+1}$ be sets in $\mathbf{R}^{d}$ in general position with respect to 0 , each containing 0 in its convex hull. Then there are at least $\frac{1}{5} d(d+1)$ colorful covering simplices.

We could get a constant little better than $\frac{1}{5}$, but since we have no reason to believe that an optimal constant could be obtained by our approach, we prefer simplicity of the numbers appearing in the proof.

Deza et al. [4] show that for $d=2$ the smallest possible number of colorful simplices is 5 , and for $d=3$ this number is either 8 or 10 . The following theorem shows that the number is 10 .

Theorem 1.3 Under the assumptions of Theorem 1.2, the number of colorful covering simplices is at least $3 d$ if $d \geq 3$. For $d=3$, the smallest possible number of colorful covering simplices equals 10.

\section{Preparations}

From now on, we assume that $X_{1}, \ldots, X_{d+1} \subset \mathbf{R}^{d}$ are $(d+1)$-point sets in general position with respect to 0 and with $0 \in \operatorname{conv}\left(X_{i}\right)$ for all $i$. We may also assume that all points of $X$ lie on the unit sphere $S^{d-1}$ (if not, we replace $X$ by its central projection on $S^{d-1}$, which affects neither the assumptions nor the conclusions of our theorems).

Every $d$-point subset $A \subset X$ generates the convex cone

$$
\operatorname{pos}(A)=\left\{\sum_{a \in A} t_{a} a: t_{a} \geq 0 \text { for all } a \in A\right\} .
$$

We let $\sigma(A)=\operatorname{pos}(A) \cap S^{d-1}$ be the corresponding spherical simplex spanned by $A$. By the general position assumption, each such spherical simplex is contained in an open hemisphere. 
The set $X_{d+1}$, the points of the last color, will play a special role in our arguments. We let $Y=X \backslash X_{d+1}$ be the subset made of the first $d$ colors, and we let $P=-X_{d+1}$ be the points antipodal to the last color class.

A transversal is any subset $T \subset Y$ with $\left|T \cap X_{i}\right|=1$ for all $i=1,2, \ldots, d$, and a partial transversal is any subset of a transversal. Let $\mathcal{T}^{d}(Y)$ denote the system of all transversals of $Y$, and for $Y^{\prime} \subseteq Y$, we let $\mathcal{T}^{d}\left(Y^{\prime}\right)=\left\{T \in \mathcal{T}^{d}(Y): T \subseteq Y^{\prime}\right\}$.

If $a \in S^{d-1}$ is a point and $T \in \mathcal{T}^{d}(Y)$, we say that $T$ covers $a$ if $a \in \sigma(T)$. Similarly, if $\mathcal{F} \subseteq \mathcal{T}^{d}(Y)$ is a system of transversals, we say that $\mathcal{F}$ covers $a$ if at least one $S \in \mathcal{F}$ covers $A$.

Colorful covering simplices, the objects of interest in Theorem 1.2, are in one-to-one correspondence with ordered pairs $(p, T)$, where $p \in P, T \in \mathcal{T}^{d}(Y)$, and $T$ covers $p$. Indeed, for any $\operatorname{such}(p, T)$, it is easily seen that $T \cup\{-p\}$ defines a colorful covering simplex (and it is equally easy to see that the correspondence is bijective, but we won't actually need that). So we aim at bounding the number of such pairs $(p, T)$ from below.

We will use the following stronger version of the colorful Carathéodory theorem [1].

Theorem 2.1 Let $X_{1}, X_{2}, \ldots, X_{d}$ be finite sets in $\mathbf{R}^{d}$ such that $0 \in \operatorname{conv}\left(X_{i}\right)$ for all $i=1,2, \ldots, d$ and let $x \in \mathbf{R}^{d}$ be arbitrary. Then there exists a d-point set $S \subseteq X_{1} \cup \cdots \cup X_{d}$ with $\left|X_{i} \cap S\right|=1$ for each $i$ and such that $0 \in \operatorname{conv}(S \cup\{x\})$.

This theorem clearly implies that the set of transversals $\mathcal{T}^{d}(Y)$ covers every point of the unit sphere, and in particular, it shows that the number of colorful simplices is at least $d+1$. We will actually apply the following consequence:

Corollary 2.2 For every point $y \in Y$ there is $p \in P$ and a transversal $T \in$ $\mathcal{T}^{d}(Y)$ that contains $y$ and covers $p$.

Proof. If $y$ is in $X_{i}$, say, then apply Theorem 2.1 to the sets $X_{j}, j \neq i$ and to the point $y$. Then $0 \in \operatorname{conv}(S \cup\{y\})$ for a suitable $S$. Setting $x=S \cap X_{d+1}$, $T=S \backslash\{x\} \cup\{y\}$ is a transversal in $\mathcal{T}^{d}(Y)$. It is easy to see that $T$ covers $-x$, which is a point in $P$.

We will also use the following lemma, with an easy topological proof.

Lemma 2.3 (Octahedron lemma) Let $S, T$ be two disjoint transversals, and let $x$ be a point covered by $S$.

(i) (Degree-0 case) If $\mathcal{T}^{d}(S \cup T)$ doesn't cover all of $S^{d-1}$, then there exists $T^{\prime} \in \mathcal{T}^{d}(S \cup T), T^{\prime} \neq S$, that also covers $x$.

(ii) (Degree-1 case) If $S$ is the unique transversal in $\mathcal{T}^{d}(S \cup T)$ covering $x$, then $\mathcal{T}^{d}(S \cup T)$ covers all of $S^{d-1}$.

The second half of the lemma is from Deza et al. [4].

Proof. We let $Q$ denote the boundary of the octahedron $\operatorname{conv}\left\{ \pm e_{1}, \ldots, \pm e_{d}\right\}$, where $e_{1}, \ldots, e_{d}$ form the standard basis of $\mathbf{R}^{d}$. We define a map $f: Q \rightarrow \mathbf{R}^{d}$ as follows. First, for all $i$, we set $f\left(e_{i}\right)=s_{i}$ and $f\left(-e_{i}\right)=t_{i}$ where $s_{i}$ and $t_{i}$ 
is the point of $S$, resp. $T$ of color $i$. Now $f$ is defined on the vertices of the octahedron, so we can extend it simplicially to its faces (which are simplices). We claim that $0 \notin f(Q)$. Indeed, $0 \in f(Q)$ would mean that 0 belongs to the convex hull of a transversal, contrary to the general position hypothesis.

Now we define another map $g: Q \rightarrow S^{d-1}$ : for $q \in Q$ let $g(q)=f(q) /\|f(q)\|$ be the projection from the origin of $f(q)$ to $S^{d-1}$. We note that $y \in S^{d-1}$ is covered by a transversal $\left\{s_{i_{1}}, \ldots, s_{i_{k}} t_{i_{k+1}}, \ldots, t_{i_{d}}\right\}$ if and only if $y$ belongs to the $g$-image of the facet

$$
\operatorname{conv}\left\{e_{i_{1}}, \ldots, e_{i_{k}},-e_{i_{k+1}}, \ldots,-e_{i_{d}}\right\} .
$$

The map $g$ has degree zero in case (i), so if it attains the value $x$ somewhere, then it has to attain it once more. In case (i) $g$ has degree 1 , and so its image covers all of $S^{d-1}$.

\section{Proof of Theorem 1.2}

Let $Y=X_{1} \cup \cdots \cup X_{d}$ and $P=-X_{d+1}$ be as in the previous section. For every $p \in P$, let $k(p)$ be the number of transversals $T \in \mathcal{T}^{d}(Y)$ that cover $p$. We thus want to bound $K:=\sum_{p \in P} k(p)$ from below.

Let $k_{\min }=\min _{p \in P} k(p)$. If $k_{\text {min }} \geq \frac{1}{5}(d+1)$, then $K \geq|P| \cdot \frac{1}{5}(d+1)>$ $\frac{1}{5} d(d+1)$, and the conclusion of Theorem 1.2 holds. So from now on, we assume $k_{\min }<\frac{1}{5}(d+1)$.

We let $p_{0} \in P$ be one of the points covered by exactly $k_{\text {min }}$ times by $\mathcal{T}^{d}(Y)$. Let $\mathcal{I}_{0} \subseteq \mathcal{T}^{d}(Y)$ consist of the $k_{\text {min }}$ transversals covering $p_{0}$, and let $Z=Y \backslash \bigcup \mathcal{T}_{0}$ be the points of $Y$ not contained in any transversals of $\mathcal{T}_{0}$ (here we mean points that are elements of the transversals, considered as finite sets, not points covered by the transversals). Let $Z_{i}=X_{i} \cap Z$. Since $\left|\mathcal{T}_{0}\right| \leq \frac{1}{5}(d+1)$, we have $\left|Z_{i}\right| \geq \frac{4}{5}(d+1)$ for all $i$.

A key to producing many transversals that cover points of $P$ is the following lemma (also see Fig. 1 for an illustration).

Lemma 3.1 (Many associated transversals) Suppose that $p \in P$ is a point covered by fewer than $\frac{1}{5} d(d+1)$ transversals of $X$, and let $S \in \mathcal{T}^{d}(X) \backslash \mathcal{T}_{0}$ be a transversal that covers $p$ but doesn't cover $p_{0}$. Let us denote by $s_{i}$ the point of $S$ of color $i$. Then there is a color $i \in\{1,2, \ldots, d\}$ and a subset $A_{S} \subseteq Z_{i} \cup\left\{s_{i}\right\}$ of at least $\frac{1}{3}(d+1)$ points such that for every $a \in A_{S}$, the transversal $S^{a}=\left(S \backslash\left\{s_{i}\right\}\right) \cup\{a\}$ also covers $p$.

Let us set $W=Z \backslash S$. For every transversal $T \in \mathcal{T}^{d}(W)$, we can apply the octahedron lemma, case (ii) (Lemma 2.3) to $S$ and $T$ with $x=p$. Indeed, no $T^{\prime} \in \mathcal{T}^{d}(S \cup T)$ can cover $p_{0}$, since $S \notin \mathcal{T}_{0}$ and $T$ is disjoint from all transversals in $\mathcal{T}_{0}$. Hence we get that there is $T^{\prime} \in \mathcal{T}^{d}(S \cup T)$ different from $S$ and covering $p$. Let us fix one such $T^{\prime}$, and let us put $U(T)=T^{\prime} \backslash S$.

Let us consider the set system $\mathcal{U}_{0}=\left\{U(T): T \in \mathcal{T}^{d}(W)\right\}$. For $U \in \mathcal{U}_{0}$, let $\bar{U}^{S}$ be the (unique) transversal $T^{\prime}$ with $U=T^{\prime} \backslash S$. The following two properties of $\mathcal{U}_{0}$ are clear from the construction. 


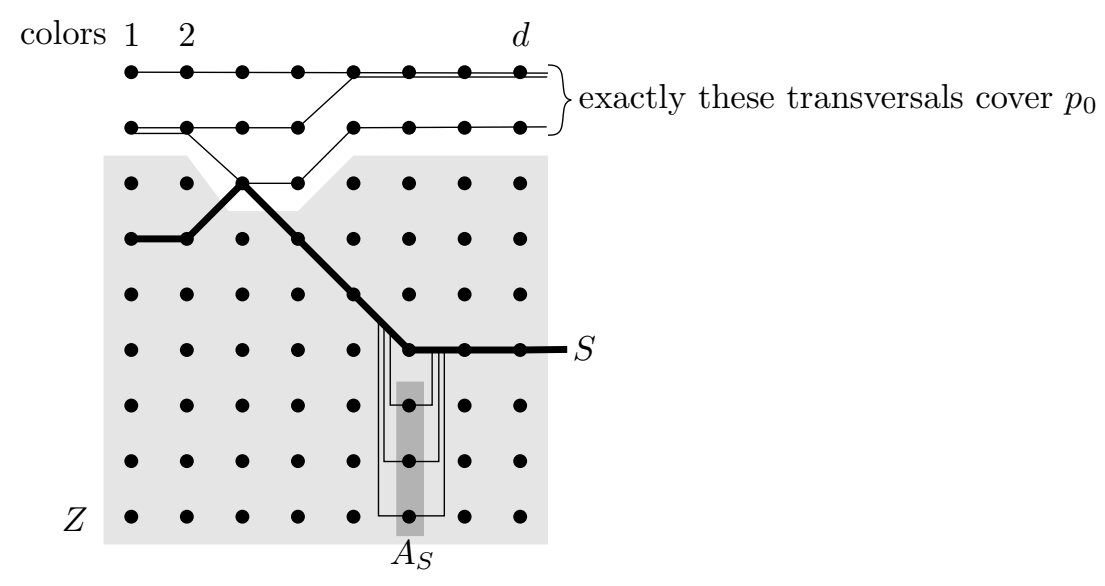

Figure 1: Illustration to Lemma 3.1.

(U1) Every $U \in \mathcal{U}_{0}$ is a nonempty partial transversal of $W$ such that $\bar{U}^{S}$ covers $p$.

(U2) Every transversal $T \in \mathcal{T}^{d}(W)$ contains some $U \in \mathcal{U}_{0}$.

Now we will delete some sets from $\mathcal{U}_{0}$ so that we obtain a system $\mathcal{U}$ still satisfying (U1) and (U2) but minimal with respect to (U2); that is:

(U3) For every $U \in \mathcal{U}$ there exists $T \in \mathcal{T}^{d}(W)$ (a "reason of existence" of $U$ ) that contains $U$ but no other set of $\mathcal{U}$.

The deletion procedure works as follows. We begin with $\mathcal{U}_{0}$ as the current system. If $U$ is a set in the current system such that every $T \in \mathcal{T}^{d}(W)$ containing it also contains some other set of the current system, we delete $U$, and we repeat this step as long as we can. The resulting system $\mathcal{U}$ satisfies all of (U1)-(U3).

Each $U \in \mathcal{U}$ corresponds to the transversal $\bar{U}^{S}$ covering $p$, so by the assumption of the lemma we have $|\mathcal{U}|<\frac{1}{5} d(d+1)$.

In order to prove the lemma, it suffices to show that there is an $i$ such that at least $\frac{1}{3}(d+1)-1$ points in $W_{i}=X_{i} \cap W$ form singleton sets in $\mathcal{U}$. Indeed, then the points of $W_{i}$ covered by singletons in $\mathcal{U}$ plus the point $s_{i}$ form the desired $A_{S}$.

First we observe that for every $i$, we have either $W_{i} \subseteq \cup \mathcal{U}$, or $W_{i} \cap \bigcup \mathcal{U}=\emptyset$. Indeed, let $U \in \mathcal{U}$ contain a point $w \in W_{i}$, and let $T \supseteq U$ be a "reason of existence" of $U$ as in (iii) above. Then $R=T \backslash\{w\}$ contains no set of $\mathcal{U}$, and hence every $T^{\prime}=R \cup\left\{w^{\prime}\right\} \in \mathcal{T}^{d}(W)$, where $w^{\prime} \in W_{i}$, has to contain some $U^{\prime} \in \mathcal{U}$ with $w^{\prime} \in U^{\prime}$.

Let $I=\left\{i \in\{1,2, \ldots, d\}: W_{i} \subseteq \cup \mathcal{U}\right\}$ be the colors covered by $\mathcal{U}$. Let $V_{i}$ be the part of $W_{i}$ not covered by singleton sets of $\mathcal{U}$, and let $n_{i}=\left|V_{i}\right|$. It suffices to show that $n_{i} \leq \frac{7}{15}(d+1)$ for some $i$, since then at least $\left|W_{i}\right|-\left|V_{i}\right| \geq$ $\frac{4}{5}(d+1)-1-\frac{7}{15}(d+1)>\frac{1}{3}(d+1)-1$ elements of $W_{i}$ are covered by singletons as needed. So we assume $n_{i}>\frac{7}{15}(d+1)$ for all $i \in I$. 
There are $M=\prod_{i \in I} n_{i}$ transversals of $V=\bigcup_{i \in I} V_{i}$ (here the transversals have $|I|$ points and they cover only the colors in $I$ ). Any $U \in \mathcal{U}$ contained in $V$ has at least two elements (since all singletons have been removed), and hence the number of transversals of $V$ containing it is

$$
\frac{M}{\prod_{i: U \cap V_{i} \neq \emptyset} n_{i}}<\frac{M}{\left(\frac{7}{15}(d+1)\right)^{2}} .
$$

Since every transversal of $V$ contains some $U \in \mathcal{U}$, we get $|\mathcal{U}| \geq\left(\frac{7}{15}(d+1)\right)^{2} \geq$ $\frac{1}{5} d(d+1)$, contradicting the assumption $|\mathcal{U}|<\frac{1}{5} d(d+1)$. This finishes the proof of Lemma 3.1.

Now we are ready to finish the proof of Theorem 1.2. For every point $z \in Z$, Corollary 2.2 guarantees the existence of a transversal $S=S(z) \in \mathcal{T}^{d}(X)$ that contains $z$ and covers some $p=p(z) \in P$. For each such $S(z)$, we apply Lemma 3.1 (of course, we may assume that no $p \in P$ is covered by more than $\frac{1}{5} d(d+1)$ transversals, since otherwise we are done). This yields the system of at least $\frac{1}{3}(d+1)$ transversals $S(z)^{a}, a \in A_{S(z)}$, that all cover $p$ and differ from $S(z)$ in at most one point. Let us denote this system by $\mathcal{A}(S(z))$ and call it the system of associated transversals of $S(z)$.

Let us put $\mathcal{S}=\{S(z): z \in Z\}$, and let $\left(S_{1}, S_{2}, \ldots, S_{t}\right)$ be an enumeration of all sets in $\mathcal{S}$ in some arbitrary order (each set occurs only once in the sequence, although the same set may be obtained for many different $z$ ).

We observe that if $\left|S_{i} \triangle S_{j}\right|>2$ (with $\triangle$ denoting the symmetric difference), then $\mathcal{A}\left(S_{i}\right)$ and $\mathcal{A}\left(S_{j}\right)$ have no transversal in common. Indeed, if both $T \in \mathcal{A}\left(S_{i}\right)$ and $T \in \mathcal{A}\left(S_{j}\right)$, then $\left|T \triangle S_{i}\right| \leq 1$ and $\left|T \triangle S_{j}\right| \leq 1$, and hence $\left|S_{i} \triangle S_{j}\right| \leq 2$. Moreover, since all $S_{i}$ have the same size, $\left|S_{i} \backslash S_{j}\right| \geq 2$ implies $\left|S_{i} \triangle S_{j}\right|>2$.

Let us call an index $i \in\{1,2, \ldots, t\}$ a jump if $\left|S_{i} \backslash S_{j}\right| \geq 2$ for every $j<i$, and a non-jump otherwise.

If $i$ is a non-jump, then $S_{i}$ adds at most one point not covered by the union $\bigcup_{j<i} S_{j}$. For a jump, $S_{i}$ may add up to $d$ points. If $J$ denotes the number of jumps and $N$ the number of non-jumps, we have $J+d N \geq|Z| \geq \frac{4}{5} d(d+1)$ (since the $S_{i}$ cover $Z$ ). Now if $J \geq \frac{1}{5} d(d+1)$, we are done since $t \geq J$ and each $S_{i}$ is a transversal covering some point of $P$. Otherwise, we have $N \geq \frac{3}{5}(d+1)$. By the above observation, the systems $\mathcal{A}\left(S_{i}\right)$ for all jumps $i$ are disjoint and each contains at least $\frac{1}{3}(d+1)$ transversals, so altogether we have at least $\frac{3}{5}(d+1) \cdot \frac{1}{3}(d+1)>\frac{1}{5} d(d+1)$ transversals. Theorem 1.2 is proved.

\section{Proof of Theorem 1.3}

We use the same notation as before. We begin with a simple lemma about a set system. We let $V_{1}, \ldots, V_{d}$ be disjoint finite sets, we set $n_{i}=\left|V_{i}\right|=n_{i}$, and we assume $1 \leq n_{1} \leq \ldots \leq n_{d}$. As before, $\mathcal{T}^{d}(V)$ denotes the set of all transversals $S$ of $V=V_{1} \cup \cdots \cup V_{d}$; that is, $S \subset V$ with $\left|S \cap V_{i}\right|=1$ for all $i$. Finally, let $\mathcal{U}$ be a system of partial transversals of $V$ satisfying conditions (U1)-(U3) as in in the proof of Lemma 3.1.. 


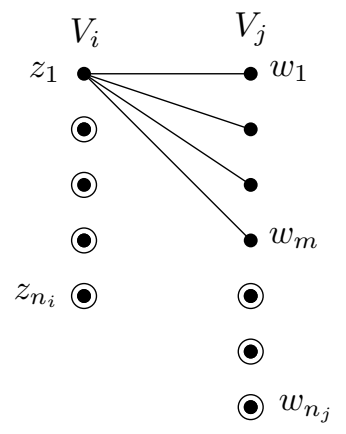

Figure 2: The set system $\mathcal{U}\left(V_{i}, V_{j}, m\right)$.

One example of such a $\mathcal{U}$ consists of all the singletons of some $V_{i}$. We denote this system by $\mathcal{U}\left(V_{i}\right)$. Another example is the following (Fig. 2): Writing $V_{i}=\left\{z_{1}, \ldots, z_{n_{i}}\right\}$ and $V_{j}=\left\{w_{1}, \ldots, w_{n_{j}}\right\}, i \neq j$, and choosing an integer $m \in\left\{1,2, \ldots, n_{j}\right\}$, we set

$$
\mathcal{U}\left(V_{i}, V_{j}, m\right)=\left\{\left\{z_{2}\right\}, \ldots,\left\{z_{n_{i}}\right\},\left\{z_{1}, w_{1}\right\}, \ldots,\left\{z_{1}, w_{m}\right\},\left\{w_{m+1}\right\}, \ldots,\left\{w_{n_{j}}\right\}\right\} .
$$

We note that $\left|\mathcal{U}\left(V_{i}, V_{j}, m\right)\right|=n_{i}+n_{j}-1$.

Lemma 4.1 Under the above conditions $|\mathcal{U}| \geq n_{1}$, with equality if and only if $\mathcal{U}=\mathcal{U}\left(V_{i}\right)$ for some $i$ with $n_{i}=n_{1}$. Moreover, if $\mathcal{U}$ is distinct from each $\mathcal{U}\left(V_{i}\right)$, then $|\mathcal{U}| \geq n_{1}+n_{2}-1$ with equality if and only if $\mathcal{U}=\mathcal{U}\left(V_{i}, V_{j}, m\right)$ for some $i, j$ with $\left\{n_{i}, n_{j}\right\}=\left\{n_{1}, n_{2}\right\}$ and some $m$ (with a suitable numbering of the points of $V_{i}$ and $\left.V_{j}\right)$.

Proof. The first statement follows easily from the fact that $\mathcal{T}^{d}(V)$ contains $n_{1}$ disjoint transversals.

For the second statement we delete all singletons $\{v\}$ from $\mathcal{U}$, and with every deleted $\{v\}$ we also delete $v$ from the ground set $V$. The remaining system $\mathcal{U}^{*}$ satisfies properties (U1), (U2), and (U3) on the remaining ground set $V_{1}^{*}, \ldots, V_{d}^{*},\left|V_{i}^{*}\right|=n_{i}^{*}$. No $V_{i}^{*}$ is empty and the total number of transversals in $\mathcal{T}^{d}\left(V^{*}\right)$ is $M=\prod n_{k}^{*}$. We also note that each $U \in \mathcal{U}^{*}$ has at least two elements.

We fix $U \in \mathcal{U}^{*}$ with $U=\left\{z_{1}, w_{1}, \ldots\right\}$, where $z_{1} \in V_{i}$ and $w_{1} \in V_{j}$. Such a $U$ is contained in at most

$$
\frac{M}{\prod_{k: U \cap V_{k}^{*} \neq \emptyset} n_{k}^{*}} \geq \frac{M}{n_{i}^{*} n_{j}^{*}}
$$

transversals. It follows that $\left|\mathcal{U}^{*}\right| \geq \min n_{i}^{*} n_{j}^{*}$, where the minimum is taken over all pairs $i, j, i \neq j$. We observe that $n_{i}^{*} n_{j}^{*} \geq n_{i}^{*}+n_{j}^{*}-1$, with equality if and only if $n_{i}^{*}=1$ or $n_{j}^{*}=1$. Adding back the deleted singletons, we get $|\mathcal{U}| \geq \min _{i \neq j} n_{i}+n_{j}-1$, and if equality holds, then either $n_{i}^{*}=1$ or $n_{j}^{*}=1$. It is not hard to check the precise conditions for equality. We omit the details.

Now we can start the proof of Theorem 1.3. If $k_{\min } \geq 3$, then we even have $3(d+1)$ colorful covering simplices. It follows from Theorem 2.1 that $k_{\min }>0$. So we have $k_{\min }=1$ or $k_{\min }=2$, and we consider these two cases separately. 
Case 1: $k_{\min }=1$. Let $p_{0} \in P$ be a point covered by a single transversal $S \in$ $\mathcal{T}^{d}(Y)$, and let $p \in S^{d-1}$ be a point in not covered by $S$. We may assume that $S=\left\{e_{1}, \ldots, e_{d}\right\}$, with $e_{1}, \ldots, e_{d}$ the standard basis of $\mathbf{R}^{d}$, because the problem is invariant under nondegenerate linear transformations. So a coordinate system is introduced. For a vector $x \in \mathbf{R}^{d}$ we write $x[j]$ for its $j$ th coordinate.

The octahedron lemma, case (ii), says that, for every $T \in \mathcal{T}^{d}(Y)$ disjoint from $S$, the set $\mathcal{T}^{d}(S \cup T)$ contains a transversal, to be denoted by $T^{\prime}$, covering $p$. We write $U(T)=T^{\prime} \backslash S$ and we set $\mathcal{U}_{0}=\left\{U(T): T \in \mathcal{T}^{d}(Z)\right\}$, where $Z=Y \backslash S$. Next we take, in the same way as in the proof of Lemma 3.1, a minimal subsystem $\mathcal{U} \subset \mathcal{U}_{0}$. The new system $\mathcal{U}$ satisfies conditions (U1)-(U3). Note that the systems $\mathcal{U}_{0}$ and $\mathcal{U}$ depend on $p$ and $S$, and so in case of need we will write $\mathcal{U}=\mathcal{U}(p ; S)$.

Claim 4.2 If $|\mathcal{U}|=d$, then $p$ has one negative coordinate and $d-1$ positive coordinates.

Proof. Lemma 4.1 shows in this case that $\mathcal{U}=\mathcal{U}\left(Z_{i}\right)$ for some $i$. For simpler notation we assume $\mathcal{U}=\mathcal{U}\left(Z_{1}\right)$, and $X_{i}=\left\{e_{1}, z_{1}, \ldots, z_{d}\right\}$.

We recall that $\sigma(T)$ denotes $S^{d-1} \cap \operatorname{pos}(T)$. For $T=\left\{x_{1}, \ldots, x_{d}\right\}$ we will also use $\sigma\left(x_{1}, \ldots, x_{d}\right)$ to denote $\sigma(T)$. Since $\mathcal{U}=\mathcal{U}\left(Z_{1}\right)$, we have $p \in \sigma\left(z_{i}, e_{2}, \ldots, e_{d}\right)$ for every $i=1,2, \ldots, d$.

Let us suppose that $p[1]>0$. Then, noticing that $\mathcal{U}=\mathcal{U}\left(Z_{1}\right)$ means $p \in$ $\sigma\left(z_{i}, e_{2}, \ldots, e_{d}\right)$ for every $i=1,2, \ldots, d$, we get $z_{i}[1]>0$ for all $i$. Consequently, $X_{1}=\left\{e_{1}, z_{1}, \ldots, z_{d}\right\}$ lies in the halfspace $\left\{x \in \mathbf{R}^{d}: x[1]>0\right\}$, contradicting the assumption $0 \in \operatorname{conv}\left(X_{1}\right)$. Since $p[1]=0$ is impossible by the general position hypothesis, we have $p[1]<0$.

A similar argument shows that $p[j]>0$ for all $j>1$. Indeed, if $p[2]<0$ (say), then $p \in \sigma\left(z_{i}, e_{2}, \ldots, e_{d}\right)$ implies $z_{i}[2]<0$ for all $i$, and then $X_{1}$ would lie in the halfspace $\left\{x \in \mathbf{R}^{d}: x[2]<0\right\}$, which is again impossible.

We recall that $k(p)$ denotes the number of transversals covering $p$. We want to show that $K=\sum_{p \in P} k(p) \geq 3 d$.

Subcase 1a: $k(p)>1$ for at least two $p \in P$. Then

$$
K \geq 2 d+(d+1-2)=3 d-1 .
$$

So $K \geq 3 d$ unless equality holds here. If equality holds, then there are exactly two points, $p_{d}, p_{d-1} \in P$ (say) with $k(p)>1$, and

$$
\left|\mathcal{U}\left(p_{d}, S\right)\right|=\left|\mathcal{U}\left(p_{d-1}, S\right)\right|=d .
$$

By Claim 4.2 both $p_{d}$ and $p_{d-1}$ have one negative coordinate and $d-1$ positive coordinates. Since $d \geq 3$, there is a coordinate $j$ with $p_{d}[j]>0$ and $p_{d-1}[j]>$ 0 . But since $k(p)=1$ implies that all coordinates of $p$ are positive (that is, $p \in \sigma(S)$ ), we have that $P$ lies completely in the halfspace $x[j]>0$, which is impossible because $0 \in \operatorname{conv}(P)$.

Subcase $1 \mathrm{~b}$ : $k(p)>1$ for exactly one $p \in P$, say for $p_{d} \in P$. Then all other $p \in P$ lie in $\sigma(S)$, and $0 \in \operatorname{conv}(P)$ implies $p_{d}[j]<0$ for all $j$. Now Claim 4.2 shows 
that $\left|\mathcal{U}\left(p_{d}, S\right)\right|=d$ is impossible. Then Lemma 4.1 yields that $\left|\mathcal{U}\left(p_{d}, S\right)\right| \geq$ $2 d-1$. Thus $k\left(p_{d-1}\right) \geq 2 d-1$ and

$$
K \geq(2 d-1)+d=3 d-1 .
$$

So $K \geq 3 d$ unless equality holds throughout: $\left|\mathcal{U}\left(p_{d}, S\right)\right|=2 d-1$ and $\mathcal{U}\left(p_{d}, S\right)$ is of the type $\mathcal{U}\left(V_{i}, V_{j}\right)$. For simpler notation we assume it is equal to $\mathcal{U}\left(V_{1}, V_{2}, m\right)$ with $X_{1}=\left\{e_{1}, z_{1}, \ldots, z_{d}\right\}$ and $X_{2}=\left\{e_{2}, w_{1}, \ldots, w_{d}\right\}$, and

$$
\mathcal{U}\left(p_{d}, S\right)=\left\{\left\{z_{2}\right\}, \ldots,\left\{z_{n_{i}}\right\},\left\{z_{1}, w_{1}\right\}, \ldots,\left\{z_{1}, w_{m}\right\},\left\{w_{m+1}\right\}, \ldots,\left\{w_{n_{j}}\right\}\right\} .
$$

Now $p_{d} \in \sigma\left(z_{i}, e_{2}, \ldots, e_{d}\right)$ implies $z_{i}[j]<0$ for all $i, j$. Also, $\sigma\left(w_{i}, e_{2}, \ldots, e_{d}\right)$ contains $p_{d}$ for $i>m$ which implies that all coordinates of $w_{i}$ are negative for $i>m$. Further, $z_{1}[j]>0$ for all $j>1$ since $z_{1}[j]<0$ for some $j>1$ would imply that $X_{1}$ lies in the halfspace $x[j] \leq 0$, and this would contradict $0 \in \operatorname{conv}\left(X_{1}\right)$, by the general position hypothesis. Now $p \in \sigma\left(z_{1}, w_{i}, e_{3}, \ldots, e_{d}\right)$ holds for $i \leq m$ which yields $w_{i}(3)<0$ for all $i \leq m$. But then $X_{2}$ lies in the halfspace $x(3) \leq 0$ which is impossible.

So we have $K \geq 3 d$ in Case 1 .

Case 2: $k_{\min }=2$. Let $p_{0} \in P$ be a point with $k\left(p_{0}\right)=2$. So $p_{0}$ is covered by exactly two transversals $S_{1}, S_{2} \in \mathcal{T}^{d}(Y)$. Set $Z=Y \backslash\left(S_{1} \cup S_{2}\right)$. To fix notation suppose $p_{m} \in \sigma\left(S_{1}\right) \cap \sigma\left(S_{2}\right)$ for $m \leq \ell$ and $p_{m} \notin \sigma\left(S_{1}\right) \cap \sigma\left(S_{2}\right)$ for $m>\ell$. We observe that $\ell<d$, since otherwise, $P \subset \sigma\left(S_{1}\right) \cap \sigma\left(S_{2}\right)$, which would contradict the assumption $0 \in \operatorname{conv}(P)$. For each $p_{m} \in P$ with $m>\ell$ we construct the sets $\mathcal{U}_{0}\left(p_{m}, S_{1}\right)$ and $\mathcal{U}_{0}\left(p_{m}, S_{2}\right)$ and the minimal subsystems $\mathcal{U}\left(p_{m}, S_{1}\right)$ and $\mathcal{U}\left(p_{m}, S_{2}\right)$. Lemma 4.1 shows that

$$
k\left(p_{m}\right) \geq\left|\mathcal{U}\left(p_{m}, S_{1}\right) \cup \mathcal{U}\left(p_{m}, S_{2}\right)\right| \geq\left|\mathcal{U}\left(p_{m}, S_{1}\right)\right| \geq d-1 .
$$

Thus

$$
K \geq 2 \ell+(d-1)(d-\ell)=d^{2}-(d+1) \ell+3 \ell+1 .
$$

In the range $\ell \in\{0,1, \ldots, d-1\}$, the last expression is minimized for $\ell=d-1$, which gives $K \geq d^{2}-(d+1)(d-1)+3(d-1)+1=3 d-1$.

So $K \geq 3 d$ unless equality holds here, in which case $\ell=d-1$, and $\left|\mathcal{U}\left(p_{d}, S_{1}\right)\right|=d-1$ and $\mathcal{U}\left(p_{d}, S_{1}\right)=\mathcal{U}\left(p_{d}, S_{2}\right)$. The last conditions imply that $\left|S_{1} \cap S_{2}\right|=d-1$ and $\mathcal{U}\left(p_{d}, S_{1}\right)$ is the special system consisting of singletons from Lemma 4.1. We may assume that $S_{1}=\left\{e_{1}, e_{2}, \ldots, e_{d}\right\}$ and $S_{2}=\left\{e_{1}^{*}, e_{2}, \ldots, e_{d}\right\}$ and $\mathcal{U}\left(p_{d}, S_{1}\right)=\left\{\left\{z_{2}\right\} \ldots,\left\{z_{d}\right\}\right\}$, where $X_{1}=\left\{e_{1}, e_{1}^{*}, z_{2}, \ldots, z_{d}\right\}$. In this case, of course, $p_{i} \in \sigma\left(S_{1}\right) \cap \sigma\left(S_{2}\right)$ for all $i<d$.

Here $e_{1}^{*}[1]>0$ since otherwise $p_{0} \in \sigma\left(S_{1}\right) \cap \sigma\left(S_{2}\right)$ wouldn't hold. Next $p_{0} \in \operatorname{pos}\left(S_{1}\right) \cap \operatorname{pos}\left(S_{2}\right)$ implies that, with certain positive reals $\alpha_{i}, \beta_{i}$,

$$
\alpha_{1} e_{1}+\alpha_{2} e_{2}+\ldots+\alpha_{d} e_{d}=p_{0}=\beta_{1} e_{1}^{*}+\beta_{2} e_{2}+\ldots+\beta_{d} e_{d} .
$$

By the general position assumption, $\alpha_{i} \neq \beta_{i}$ for all $i$. Thus we may assume that $e_{1}^{*}[2]<0$ (possibly swapping $S_{1}$ and $S_{2}$ ).

Now it is easy to show that $K=3 d-1$ is impossible. For each $i \geq 2, z_{i}[2]<0$ must hold since every coordinate of $p_{d}$ is negative and $p_{d} \in \sigma\left(z_{i}, e_{2}, \ldots, e_{d}\right)$ for 
each $i \geq 2$. But then $X_{1}=\left\{e_{1}, e_{1}^{*}, z_{2}, \ldots, z_{d}\right\}$ lies in the halfspace $x[2] \leq 0$, which contradicts the assumption $0 \in \operatorname{conv}\left(X_{1}\right)$.

Remark. It is perhaps interesting to note that $K \geq 3 d-1$ is much easier to prove than $K \geq 3 d$. In fact, $K \geq 3 d$ does not hold when $d=2$ and, of course, we had to use $d>2$ during the proof.

MENTION THE SET SYSTEM PROBLEM (MIN SIZE OF U SATISFYING (U1)-(U3))

\section{References}

[1] I. Bárány. A generalization of Carathéodory's theorem. Discrete Math., 40:141-152, 1982.

[2] I. Bárány and S. Onn. Carathéodory's theorem, colourful and applicable. In Intuitive Geometry (Budapest 1995), Bolyai Soc. Math. Stud. vol. 6, János Bolyai Math. Soc., Budapest, 1997, pages 11-21.

[3] I. Bárány and S. Onn. Colourful linear programming and its relatives. Math. OR (1997), 22:550-567, 1997.

[4] A. Deza, S. Huang, T. Stephen, and T. Terlaky. Colourful simplicial depth. Submitted, 2005. 